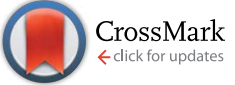

Cite this: RSC Adv., 2017, 7, 17264

\title{
Fluorescence sensing of amine vapours based on ZnS-supramolecular organogel hybrid films
}

\author{
Huiyun Xia, ${ }^{a}$ Guanyu Liu, ${ }^{a}$ Chuan Zhao, ${ }^{b}$ Xiaojuan Meng, ${ }^{a}$ Fangfang Li, ${ }^{a}$ \\ Fengyan Wang, ${ }^{a}$ Li Duan $^{a}$ and Huaxin Chen ${ }^{\star a}$
}

A stable fluorescent ZnS-supramolecular organogel hybrid film was fabricated and investigated for the sensing of volatile organic monoamine and diamine vapour. The film was prepared by placing a supramolecular organogel film incorporated with $\mathrm{Zn}(\mathrm{Ac})_{2}$ in $\mathrm{H}_{2} \mathrm{~S}$ atmosphere for $48 \mathrm{~h}$ at ambient temperature. The fabrication of the organogel film was achieved by spin-coating of the hot lowmolecular-mass gelator (LMMG) dimethylformamide solution onto the surface of a glass plate. The hybrid film exhibits uniform cross-linked nanofibres, as revealed by SEM measurements. The obtained organogel film demonstrated promising performance as a substrate for stabilizing ZnS nanoparticles loaded in the ZnS-supramolecular organogel hybrid film. The quantity of the ZnS nanoparticles embedded in the hybrid film can be conveniently adjusted by the initial concentrations of $\mathrm{Zn}^{2+}$. The sensing mechanism of the combination of static and dynamic quenching was elucidated. The sensing performances of the film for common volatile organic compounds were investigated with a detection limit of 11.0 ppm obtained for ethylenediamine vapour. It is found that the sensing response time is strongly related to the volatile performance of the organic amines tested.

Received 13th January 2017

Accepted 6th March 2017

DOI: $10.1039 / \mathrm{c} 7 \mathrm{ra00556c}$

rsc.li/rsc-advances employed a fluorescence polyfluorene/palladium tetraphenylporphyrin (PFO/PdTPP) composite film for the detection of amine vapour, and a lasing "turn-on" sensory mechanism was proposed. ${ }^{17}$ Ahn and co-workers reported that phenolcontaining bis(oxazolines) sensors showed photoluminescence (PL) enhancement towards primary amines but quenching towards secondary and branched amines. ${ }^{18}$ Recently, Swager and co-workers took fluorescent conjugated polyelectrolytes (CPEs) as sensing materials to detect spermidine, putrescine, and spermine. ${ }^{19}$ Chemical sensors capable of detecting amine species conveniently are particularly attractive. ${ }^{\mathbf{2 0 , 2 1}}$ However, so far much more progress has been obtained for the liquid phase rather than the vapour phase. ${ }^{1}$ There is an unremitting pursuit for developing highly selective and sensitive new materials towards fluorescence detection of amine vapour.

ZnS, an important II-VI group semiconductor, has many remarkable fundamental properties, such as a wide-band-gap (3.72 for cubic zinc blend) semiconductor, possessing polar surfaces, excellent transport properties, thermal stability and high electronic mobility. ${ }^{22,23}$ These unique characteristics made ZnS can form various interesting nanostructure, resulting in rich application in the electronics and sensing areas. ${ }^{22-24}$ The gas sensing performance of $\mathrm{ZnS}$ are strongly influenced by its microstructural and morphological characteristics, such as the shape, density, the size of particle, size distribution and the number of surface defects. ${ }^{25}$ Recently, considerable efforts have been committed to the preparation of $\mathrm{ZnS}$ films that can be reusable and expediently made into equipment. ${ }^{26,27}$ Various
${ }^{a}$ School of Materials Science and Engineering, Chang'an University, Xi'an 710064, P. R. China.E-mail: chenhx_paper@163.com; xiahy@chd.edu.cn

${ }^{b}$ School of Chemistry, The University of New South Wales, Sydney, NSW 2052, Australia 
preparation methods have been reported fabricating these film sensors such as sputter coating, vacuum evaporation, molecular beam epitaxy (MBE), metal organic chemical vapor deposition (MOCVD), electrochemical co-deposition and hydrothermal/ solvothermal synthesis, atomic layer epitaxy (ALE). ${ }^{28-33}$ Nevertheless, some techniques require expensive apparatus, lengthy pre-processing time and generate a great deal of wastewater.

Supramolecular gels formed by low-molecular-mass gelators (LMMGs) are of particular importance and interest due to their special properties and forming mechanisms. ${ }^{34-36}$ In the recent years, supramolecular organogels have been exploited as structure-directing agents to generate different types of nanomaterials, such as belts, tubes, rods, and balls. ${ }^{37}$ As one of the most important types of LMMGs, cholesterol (Chol) derivatives have attracted considerable attention as building block for selfassembling versatile supramolecular gels. ${ }^{38}$ Based on hydrogenbonding and van der Waals forces, various microstructures can be assembled in solutions, gel networks and solids by Chols. ${ }^{39}$ Additionally, the inside of a gel network introduced with inorganic nano-particles have been attracted great attention since a new kind of inorganic/organic nano-composite materials will be produced, which would exhibit some novel properties combing the merits of organic, inorganic and nano-materials. ${ }^{35-37}$ Nevertheless, there is no report on the sensing of inorganic nanostructure templated by supramolecular organogels, except our previous report on CdS.$^{40}$ Actually, so far, in the area of gas sensing, metal sulfides have been primarily investigated modifying with other inorganics to improve the sensing performance of the latter. ${ }^{41,42}$ The researches of metal sulfides used as sensing materials individually for gas detection is few, and have no in-depth study about their sensing properties. ${ }^{43,44}$

Taking into account the availability of a sensor and high toxicity of CdS nanoparticles ${ }^{45,46}$ nontoxic, biocompatible, chemically and photo-chemically stable ZnS was adopted as sensing materials. ${ }^{25}$ In this study, we report a Chol derivativesbased ZnS-supramolecular organogel hybrid film for amine vapour sensors, furthermore, compare with the CdS-organogel previous reported, ${ }^{40}$ the preparation method was much simpler and more environmentally friendly. It is anticipated that Chols with adjustable self-assembling properties could be used as templates for the synthesis of ZnS-organogel composites, and meantime provide cavities for capturing the analyte gas molecules. The obtained results show that the fluorescence emission of the synthesized film is very sensitive to volatile organic monoamines and diamines vapours, and the sensing response time depends on the volatile performance of the amines detected.

\section{Experimental details}

\subsection{Materials}

All chemicals used were of analytical grade. Zinc acetate dihydrate $\left(\mathrm{Zn}(\mathrm{Ac})_{2} \cdot 2 \mathrm{H}_{2} \mathrm{O}\right)$ was obtained from Yaohua (Tianjing, China) and used directly without further purification. Tetrahydrofuran (THF), $n$-hexane, dichloromethane, triethylamine (TEA), and benzene were purified before use. $N, N$-Dimethylformamide (DMF) was purified by vacuum distillation. Boc-
L-alanine, cholesterol, dicyclo-hexylcarbodiimide (DCC), $\mathrm{N}, \mathrm{N}$ dimethylamino-pyridine (DMAP), thioacetamide, and sodium hydroxide were used directly. Other reagents, including methanol, hydrochloric acid, formaldehyde, ethanol, ethylenediamine (EDA), pyridine, 1,4-butanediamine (BDA), 1,3propanediamine (PDA), aniline, 1,6-hexanediamine (HDA), $N, N$ dimethylaniline, $N$-methylaniline, acetone, butylamine, acetic acid, ammonia, and cyclohexane were used as received. The double distilled water was used throughout the work.

\subsection{Preparation of a substrate film}

The LMMGs of cholesterol derivatives were synthesized according to the literature and used as a film forming substrate,${ }^{47}$ the molecular structure of the LMMGs is shown in Scheme 1. To prepare the gel film, $1 \mathrm{~mL}$ DMF with dissolved $\mathrm{Zn}(\mathrm{Ac})_{2}$ and a series of the gelator $(0.004 \mathrm{~g}, 0.008 \mathrm{~g}, 0.0125 \mathrm{~g}$, $0.0165 \mathrm{~g}$ or $0.020 \mathrm{~g})$ were placed into sealed glass tubes $(10 \mathrm{~mm}$ i.d.) separately, then the tube was heated until the gelator dissolves totally. Finally, a certain volume of the above solution $(400 \mu \mathrm{L})$ was sucked out by a heated pipette and deposited onto a preheated and cleaned glass substrate surface. With the gradual decrease of the temperature of the glass substrate to ambient temperature, a stable organogel film containing $\mathrm{Zn}^{2+}$ was formed.

\subsection{Preparation of $\mathrm{ZnS}$-organogel hybrid films}

The obtained substrate film dissolving $\mathrm{Zn}^{2+}$ was placed in an expressly designed reaction vessel after $4 \mathrm{~h}$ of aging. Here, $\mathrm{H}_{2} \mathrm{~S}$ was produced by the hydrolysis of thioacetamide, the mixture of $7 \mathrm{mM}$ of thioacetamide and $3 \mathrm{~mL}$ of $\mathrm{HCl}$ solution $\left(3 \mathrm{~mol} \mathrm{~L}^{-1}\right)$ placed in a culture dish were put in the bottom of the above reaction vessel. Then, clear $\mathrm{H}_{2} \mathrm{~S}$ was diffused gradually into the reactor for $48 \mathrm{~h}$. The redundant $\mathrm{H}_{2} \mathrm{~S}$ was removed by evaporation under a vacuum pump for $6 \mathrm{~h}$ to obtain ZnS-organogel hybrid films. Herein, the amount of ZnS deposited in the films could be adjusted by changing the initial concentrations of $\mathrm{Zn}^{2+}$, which were $1.0 \times 10^{-1}, 1.0 \times 10^{-2}, 1.0 \times 10^{-3}, 1.0 \times 10^{-4}$ or $1.0 \times 10^{-5} \mathrm{~mol} \mathrm{~L}^{-1}$, respectively. It is noteworthy that the morphology and luminescent properties of the composite films did not change after preservation in a desiccator for 6 months.

\subsection{Characterization techniques}

X-ray diffraction measurement was recorded by a Japan Rigaku $\mathrm{D} /$ max-III X-ray diffractometer with $\mathrm{Cu} \mathrm{K} \alpha$ radiation at $35 \mathrm{kV}$ and $40 \mathrm{~mA}$. Diffraction pattern was recorded in the $2 \theta$ range of $15-80^{\circ}$ with $0.02^{\circ}$ steps.

Scanning electron microscopy (SEM) with energy-dispersive X-ray spectroscopy (EDX) measurements were detected on

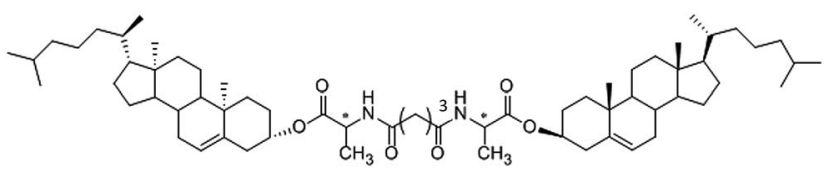

Scheme 1 The molecular structure of the LMMGs. 
a Hitachi S-4800 Field Emission Scanning Electron Microscopy Spectrometer. The gel film and $\mathrm{ZnS}$-organogel hybrid film were dried at room temperature for 1 day under normal air conditions and then evaporated by a vacuum pump at $50{ }^{\circ} \mathrm{C}$ for $12 \mathrm{~h}$. The accelerating voltage was $3 \mathrm{kV}$, and the emission was $10 \mu \mathrm{A}$. Before testing, the film was fixed to a sample holder with a conductive tape, and then it was sputtered with a thin layer of $\mathrm{Au}$.

Fluorescence spectra were performed at ambient temperature on a Perkin Elmer LS 55 Fluorescence spectrometer with the emission and excitation wavelengths of $550 \mathrm{~nm}$ and $336 \mathrm{~nm}$, respectively. PL lifetime were recorded according to standard time-correlated single photon counting fluorescence spectrometer (Edinburgh Instruments FLS 920) with the front face method. The prepared film was fixed in a quartz cuvettes with the ZnS-organogel surface toward the inside of it. It should be noted that each set of position during the measurement of the film remain unchanged.

Ultra violet-visible absorption spectroscopy were measured with a UV-3600 Shimadzu UV-vis-NIR spectrophotometer at ambient temperature. Firstly, a cleaned substrate was fixed on the surface of $\mathrm{BaSO}_{4}$ whiteboard, secondly, the spectra was scanned for background correction, then, the film sample was fixed on the surface of $\mathrm{BaSO}_{4}$ whiteboard, and finally the spectra of the samples were scanned and recorded under the same settings.

Transmission electron microscopy (TEM) was carried out on a JEOL JEM 2100F instrument. Firstly, the sample were scraped from the surface of the glass substrate carefully, then, the sample was dispersed in ethanol by ultrasonic pre-treatment for $30 \mathrm{~min}$, which is a poor solvent of supramolecular organogel, and finally a drop of the supernatant dispersion was placed on a carbon-coated copper grid. Analysis was done at $200 \mathrm{kV}$.

The size distribution of $\mathrm{ZnS}$ nanoparticles was determined by means of dynamic light scattering (DLS) with a Brookhaven Instrument. The sample was scratched from the surface of the glass substrate and dispersed in tetrahydrofuran, which is a good solvent of supramolecular organogel, the supernatant solution containing ZnS nanoparticles was measured.

\subsection{PL quenching method}

Firstly, $10 \mu \mathrm{L}$ of the solvent to be detected was added to the cuvette with the prepared film interior. Then, the cuvette was instantly sealed by sealing film. Finally, the PL emission spectrum of the hybrid film was scanned every $2 \mathrm{~min}$. The formula of $1-\left(I / I_{0}\right)$ can be determined the quenching efficiency of the films, where $I$ and $I_{0}$ represent the fluorescence intensity of the fabricated film in the presence or absence of the analyte, respectively.

\section{Results and discussion}

\subsection{Physical characterization of the sensing films}

Fig. 1a and b show the SEM images of the organogel substrate film and the ZnS-supramolecular organogel hybrid film, respectively. As shown in Fig. 1a, the dried gel film without

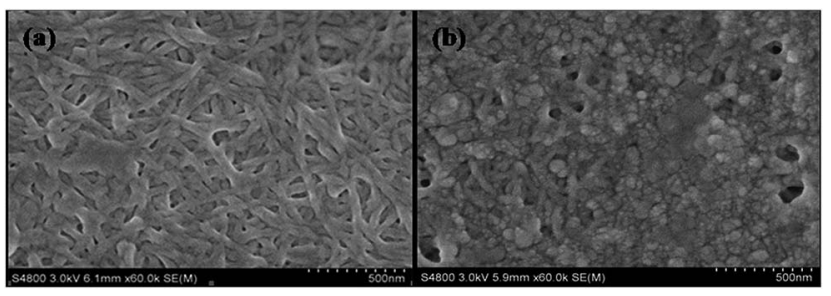

Fig. 1 SEM images of (a) the organogel substrate film and (b) ZnSsupramolecular organogel hybrid film.

solvent is composed of relatively homogeneous crosslinking fibres with the diameter of $25 \mathrm{~nm}$ approximately. This is to some degree expected as fibres are the common morphology of the aggregates of LMMG used in fabricating the organogels. ${ }^{36-38}$ The morphology of the composite film in Fig. 1b shows that spherical nanoparticles are dispersed uniformly in the interior and on the surface of the network structure.

To determine the stabilizing influence of the fibre networks on the ZnS nanoparticles produced via this method, a series of gel films with different amounts of the gelator $(0.008 \mathrm{~g}, 0.0125 \mathrm{~g}$, $0.0165 \mathrm{~g}$ or $0.02 \mathrm{~g}$ ) was adopted, as shown in Fig. 2. It is clear that by increasing the dosage of the gelator, there is a trend of increasing number of gel fibre bundles, a denser network structure, and consequently, a sharp decrease in the network channel. Further, it is demonstrated that most of the ZnS nanoparticles are deposited on the surface of the gel film when the gel film was adopted with more dosage of gelator $(0.02 \mathrm{~g})$. Therefore, the confinement effect of the gelator is less effective in the gel film with much denser fibre bundles, and subsequently the growth of nanoparticles could not be controlled efficiently. Moreover, because the hybrid film with dense network structure possesses less cavities, it is less favourable for capturing the analyte gas. Thus, as for the gas sensing, the gel films with smaller dosages of gelator is preferred in this work.

XRD was further used to analyse the crystal structure of the deposited materials. As shown in Fig. 3, three peaks in the diffraction pattern at $28.58^{\circ}, 47.82^{\circ}$ and $56.52^{\circ}$, which were separately indexed as (111), (220), and (311) of cubic ZnS phase

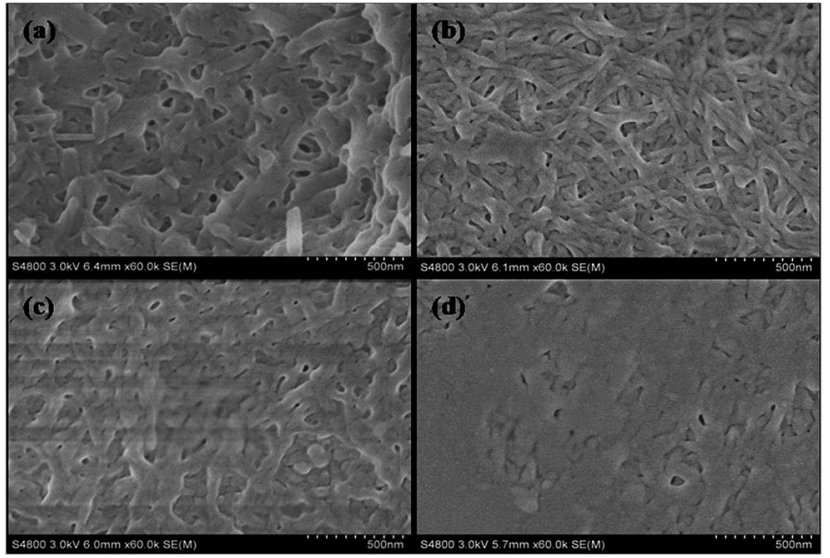

Fig. 2 SEM images of the supramolecular organogel films with $0.008 \mathrm{~g}$ (a), $0.0125 \mathrm{~g}$ (b), $0.0165 \mathrm{~g}$ (c) or $0.02 \mathrm{~g}$ (d) gelator, respectively. 


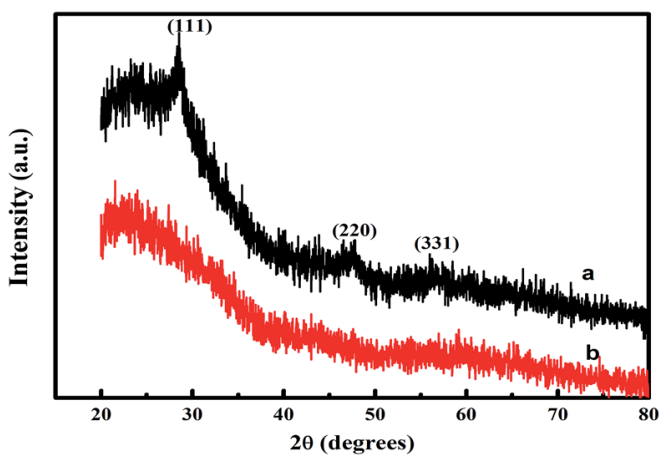

Fig. 3 X-ray diffraction patterns of (a) the ZnS-supramolecular organogel hybrid film and (b) supramolecular organogel film (the prime concentration of $\mathrm{Zn}(\mathrm{Ac})_{2}$ is $\left.0.10 \mathrm{M}\right)$.

when compared with the standard JCPDS data (file no. 19-0191). A broad reflection peak at $2 \theta$ degrees approximately $20^{\circ}$ in curve $b$ can be assigned to the presence of the gelator, which is often observed in the organics. These results indicate the $\mathrm{ZnS}$ nanoparticles are effectively deposited in the gel film and the composite film is prepared successfully.

\subsection{Fluorescence characterization of the sensing film}

As shown in Fig. 4, the normalized static fluorescence emission spectra of the ZnS-supramolecular organogel film containing different quantities of $\mathrm{ZnS}$ were taken under an excitation wavelength of $336 \mathrm{~nm}$. Compared with the background organogel containing $\mathrm{Zn}(\mathrm{Ac})_{2}$, $\mathrm{ZnS}$-organogel hybrid films has intense PL emission. It can therefore be concluded that the emission arises from $\mathrm{ZnS}$, as depicted in the illustration in Fig. 4. In addition, the PL emission spectra is broad and has no meticulous structures, suggesting that the grain size distribution of $\mathrm{ZnS}$ nanoparticles embedded in the hybrid films was relatively wide. Furthermore, with the increase of $\mathrm{ZnS}$ content, the maximum emission wavelength of the hybrid films shifts from $535 \mathrm{~nm}$ to $545 \mathrm{~nm}$. Such a wide low-energy PL spectrum is mainly due to trapped state emissions originates from surface defect sites, which is related to excess sulfur, low crystallization

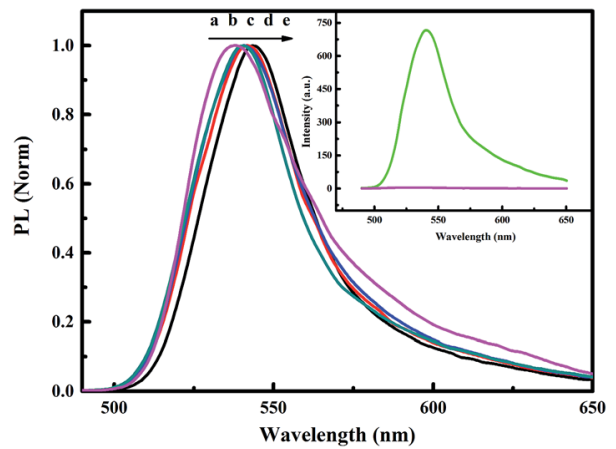

Fig. 4 Normalized photoluminescence $(\mathrm{PL})$ of the ZnS-organogel hybrid films. From (a) to (e), the initial concentration of $\mathrm{Zn}^{2+}$ is $1.0 \times$ $10^{-5}, 1.0 \times 10^{-4}, 1.0 \times 10^{-3}, 1.0 \times 10^{-2}$ and $1.0 \times 10^{-1} \mathrm{~mol} \mathrm{~L}^{-1}$, respectively $\left(\lambda_{\mathrm{ex}}=336 \mathrm{~nm}\right)$. and other interface defects of the nano-crystals. ${ }^{48-50}$ In fact, band edge and trap emissions often coexist in sulphide semiconductor nanoparticles, ${ }^{51,52}$ and by increasing the number of surface defects, the trap emission could be strengthened significantly. ${ }^{52}$ Thus, the band edge emission obtained through the recombination mechanism could be completely inhibited. Similar behaviours have been reported for the absorption and luminescence properties of the surface states in $\mathrm{ZnS}$ nanostructures. ${ }^{53}$ The absorption maximum of bulk $\mathrm{ZnS}$ at $345 \mathrm{~nm}$ is also blue shifted to $50 \mathrm{~nm}$ with the size of $\mathrm{ZnS}$ nanoparticles decreasing, as shown in Fig. 5. It has been known that with the decrease of the size of embedded ZnS particles, the wavelength of maximum emission will be blue shifted due to quantum size effects. $^{54}$

The fluorescence emission of the $\mathrm{ZnS}$-organogel film is observed red-shifted, which is consistent with the shift occurred in the UV-vis spectra ( $c f$. Fig. 5). From the figure, it can be found that as the increase of the prime concentration of $\mathrm{Zn}(\mathrm{Ac})_{2}$, the band edges of the $\mathrm{ZnS}$ nanoparticles at around $290 \mathrm{~nm}$ shift to longer wavelengths. This behaviour has been reported by others, suggesting the edge of the spectrum shifts to longer wavelength along with the growing of inorganic nanoparticles size. ${ }^{55}$

TEM image was used to investigate the size of $\mathrm{ZnS}$ particles in the hybrid film, as shown in Fig. 6a. It can be observed that ZnS nanoparticles exhibit spherical morphology and the average size of ZnS nanoparticles is about $300 \mathrm{~nm}$. Additionally, in order to study the size distribution of the $\mathrm{ZnS}$ nanoparticles, DLS was conducted ( $c f$. Fig. 6b), it is obvious that the primary formed ZnS NPs have a size of approximately $293 \mathrm{~nm}$, which is accordance with TEM measurement result.

\subsection{Sensing performance}

Selectivity is one crucial property for the sensing materials. Fig. 7 shows the histograms of $1-\left(I / I_{0}\right)$ to common volatile organic compounds. It was found that the volatile organic diamines and monoamines, for example EDA, PDA, BDA, aniline and methylamine could quench the emission significantly, furthermore, diamines exhibit stronger quenching effect than organic monoamines. However, $N, N$-dimethylaniline and

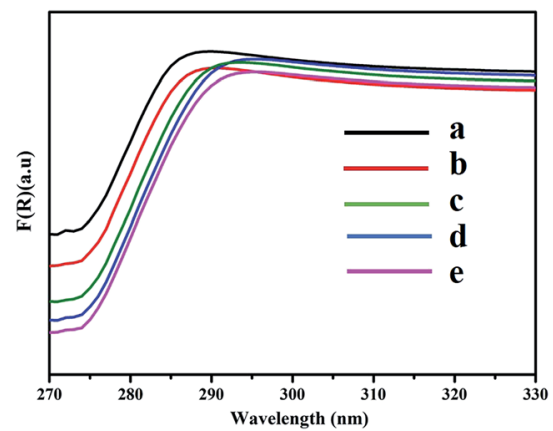

Fig. 5 The UV-vis spectra of the ZnS-organogel films with different initial concentrations of $\mathrm{Zn}(\mathrm{Ac})_{2}$. From (a) to (e), the concentration is $1.0 \times 10^{-5}, 1.0 \times 10^{-4}, 1.0 \times 10^{-3}, 1.0 \times 10^{-2}$ and $1.0 \times 10^{-1} \mathrm{~mol} \mathrm{~L}^{-1}$, respectively. 

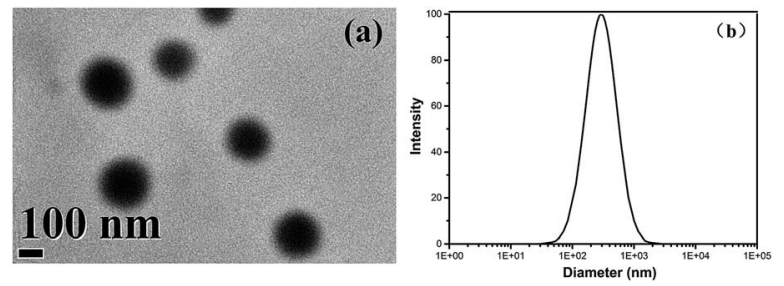

Fig. 6 TEM images (a) and DLS measurement (b) of ZnS nano particles dispersions.

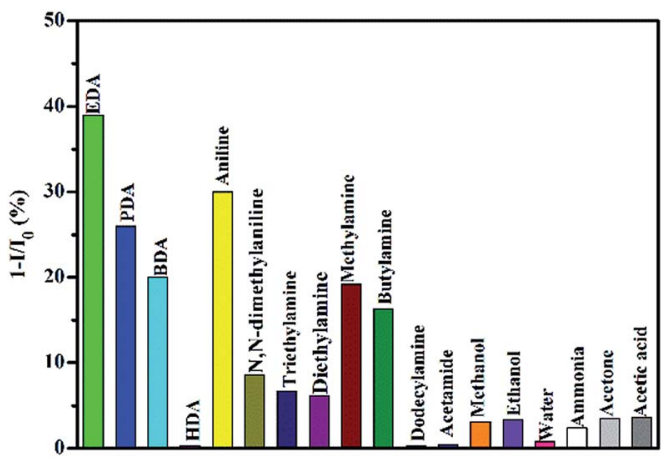

Fig. 7 The maximum quenching efficiencies of common organic reagents to the fluorescence emission of hybrid films $\left(\lambda_{\mathrm{ex}}=336 \mathrm{~nm}\right)$

butylamine show limited quenching effect with the maximum quenching efficiency of $9 \%$ and $16 \%$, respectively. Secondary and tertiary amines such as diethylamine and triethylamine exhibited a weak quenching effect. Ammonia almost has no response to the emission of the film. The quenching efficiencies for other common compounds, such as acetamide, methanol, ethanol, water, acetone and acetic acid, are less than $4 \%$. In addition to these, solid amines such as dodecylamine and 1,6hexanediamine show almost no response to the fluorescence emission of the film.

To investigate the interference effect of common compounds to the detection of amine vapours, EDA was taken as example of quenching agent, a series of experiments were designed and conducted. Firstly, $10 \mu \mathrm{L}$ of EDA was added to the cuvette, then, different amounts of EDA of acetamide were added to the above cuvette individually $(2,4,6 \mu \mathrm{L})$. Finally, the PL emission spectrum of the hybrid film was scanned every $2 \mathrm{~min}$. It is found that PL spectra of sensing film is almost the same in the presence of different content of acetamide, and are exactly the same as those in the absence of acetamide. Similarly, the interference effect of other compounds were investigated by above method, the same conclusion was obtained. So the existence of these common compounds can't interfere with the detection of amine vapours.

Considering that EDA is a popular and strong chelating agent, it was taken as an example to study the sensing performance of the hybrid films to volatile organic diamine and monoamine. ${ }^{56}$ The fluorescence emission of hybrid films in the presence of EDA at different exposing time is shown in Fig. 8a. It can be observed that the fluorescence intensity of the hybrid
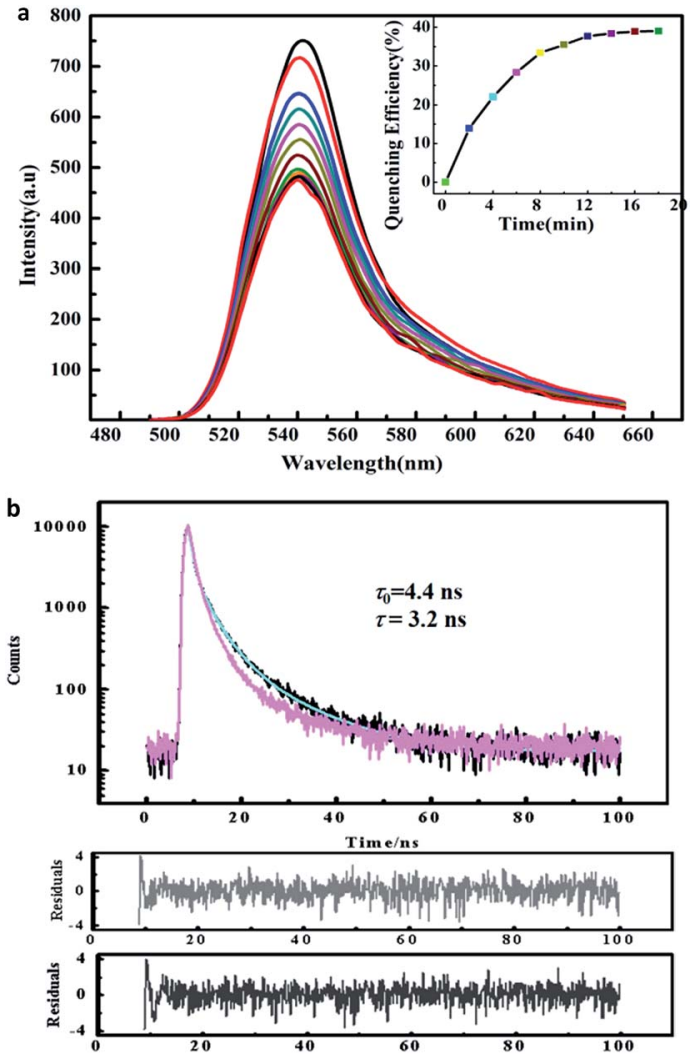

Fig. 8 (a) Fluorescence emission spectra of the sensing film in the EDA vapour at different exposure time. The insert map is the quenching efficiency curve against exposure time $\left(\lambda_{\mathrm{ex}}=336 \mathrm{~nm}\right.$ ). (b) The PL fluorescence decays of the film in the EDA vapour (the bottom) and that in the air (the top) $\left(\lambda_{\mathrm{ex}}=336 \mathrm{~nm}\right)$.

film reduced dramatically with the extension of exposure time. Furthermore, when the exposure time reaches $12 \mathrm{~min}$, approximately $36 \%$ of the emission is quenched ( $c f$. illustration of Fig. 8a). By further study of Fig. 8a, it is revealed that there are two sections during the gas quenching process, a rapid quenching process, which has led to about $36 \%$ of the emission quenching, and a very sluggish quenching process. The combination of two quenching processes suggest that some emission centers may be exposed to the outer surface of the hybrid film, making them easier to achieve. However, others may hide deeply, so it is too hard for the gas quencher to reach them. The detection limitation (DL) of the hybrid film to EDA vapor is calculated to be $11 \mathrm{ppm}$ using the formula in 57 .

Usually, the quenching mechanism includes in static quenching, dynamic quenching or a combination of the two. It is well known the static quenching has a changeless fluorescence lifetime $(\tau)$, namely, the value of $\tau$ is fixed having no relation with quencher contents. ${ }^{57,58}$ However, as for dynamic quenching, $\tau_{0} / \tau=I_{0} / I, \tau_{0}$ and $\tau$ are the fluorescence lifetimes of the fluorophore in the absence and presence of the quencher, respectively. So the sensing mechanism can be established by comparing time-resolved quenching results with steady-state quenching result. Fig. 8b shows the PL lifetime data, it is clear that the specific value of $\tau_{0} / \tau$ is 1.4 at the quenching equilibrium state, which is lower than the ratio of $I_{0} / I(1.7)$ when 
the quenching process is finished (illustration in Fig. 8a). Thus, it is suggested that the quenching process in the presence of EDA is dynamic. ${ }^{58}$ This result could be rationalized by most of the surface is covered by the sulfur after exposure to the $\mathrm{H}_{2} \mathrm{~S}$ gas, and the presence of $\mathrm{ZnS}$ nano-particles in the hybrids, making the adsorption/desorption became repeatable, which has been reported in the studies of photoluminescence decrease of CdSe nanoparticles in the presence of $n$-butylamine. ${ }^{59}$ As for the existence of static quenching, it can be rationalized by considering the sizes of ZnS nanoparticles are not completely homogeneous, due to the fact that some volatile diamines such as EDA and PDA exhibit a larger, more complex equilibrium constant in contrast with other volatile amines. ${ }^{56}$ Therefore, it is proposed that this part quenching of EDA to the fluorescence emission of hybrid film is the static quenching mechanism as a result of the formation of complex of EDA molecules with the surface trap on $\mathrm{ZnS}$ in the hybrid film. ${ }^{60}$

Taking into account the test method in the sensing experiment, it is rationalized that quenching sensitivity depends on two factors: (i) organic amine's volatilization rate, and (ii) diffusion of the organic amines from the gas phase to the interior of the hybrid film. In order to demonstrate the hypothesis, BDA was used as a quencher to conduct the same sensing measurement. BDA has a higher molecular weight and more sluggish evaporation rate compared with EDA. From the result depicted in Fig. 9, it is clear that $1-\left(I / I_{0}\right)$ of BDA is smaller than that of EDA, supporting the hypothesis that sluggish volatilization and diffusion rate lead to smaller quenching efficiency.

\subsection{Reversibility of the quenching process}

As a sensing film, the reversibility and repeatability is very crucial for the practical application. Therefore, the reversibility of the sensing process is investigated using an established procedure as follows: ${ }^{40}$ firstly, the film was fixed in the interior of a cuvette, and the florescence emission of the film was recorded. After that, 10 $\mu \mathrm{L}$ of EDA was dropped into the bottom of cuvette carefully, and the cuvette was sealed immediately. When florescence emission intensity remain stable after about $12 \mathrm{~min}$, the PL emission was measured again. Finally, the emission of the film was recovered by purging with hot air for 10 minutes using a blower at room temperature. The aforementioned quenching and recovery

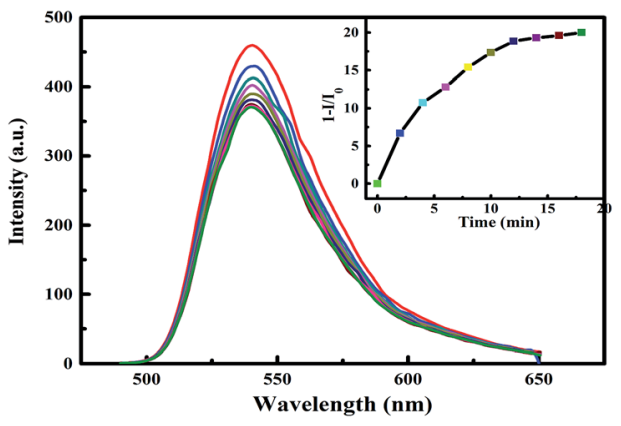

Fig. 9 Fluorescence response of the hybrid film in the BDA vapour as a function of exposure times. The illustration is the $1-\left(I-I_{0}\right)$ against exposure time $\left(\lambda_{\mathrm{ex}}=336 \mathrm{~nm}\right)$.

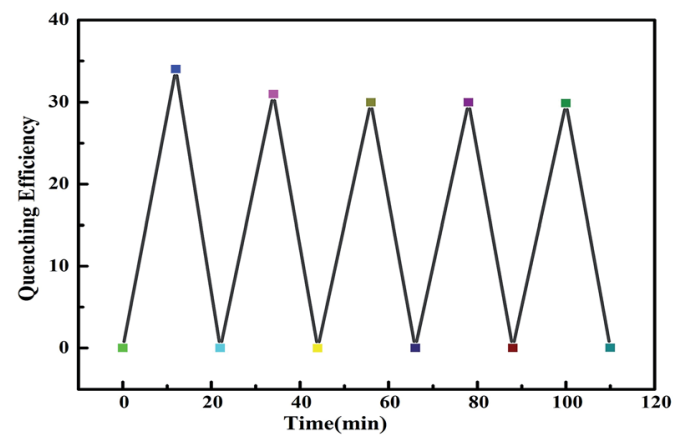

Fig. 10 The reversibility of the response of fluorescent film to the hybrid film to EDA vapour ( $\lambda_{\mathrm{ex}}=336 \mathrm{~nm}$ ).

process was repeated five times ( $c f$. Fig. 10). The response of film to the presence of EDA tends to be stable after one use.

\section{Conclusions}

In summary, a selective fluorescent ZnS-supramolecular organogel hybrid film has been constructed for sensing volatile organic monoamines and diamines vapour by adopting supramolecular gel films as substrates. The gel film offers a nanofibre morphology with abundant cavities, which is beneficial for catching analyte vapour molecules. Furthermore, the hybrid films loading various contents of $\mathrm{ZnS}$ can be prepared conveniently by adjusting the prime precursor concentration. The emission of the developed hybrid film is quenched by some volatile organic diamines and monoamines with the response rate depends distinctly on the volatile nature of the analytes tested. The film also exhibits excellent reversibility for EDA. It is expected that this kind of supramolecular organogel hybrid film could find applications for the detection of volatile organic diamines and monoamines in industry and environment.

\section{Acknowledgements}

The authors would like to thank the Natural Science Foundation of China (No. 51202016, 51502021, 51102023), Basic Scientific Research of Central Colleges, Chang'an University (No. 310831151080, 310831161015), Outstanding Youth Fund, Chang'an University (No. 310831163501). C. Z. thanks for the financial support from the Australian Research Council (DP150101861).

\section{Notes and references}

1 Y. K. Che, X. M. Yang, S. Loser and L. Zang, Nano Lett., 2008, 8, 2219-2223.

2 T. Gao, E. S. Tillman and N. S. Lewis, Chem. Mater., 2005, 17, 2904-2911.

3 Q. Wei, P. Parzuchowski, W. Zhang and M. E. Meyerhoff, Anal. Chem., 2003, 75, 332-340.

4 F. Reviriego, P. Navarro, E. Garcia-Espana, M. T. Albelda, J. C. Frias, A. Domenech, M. J. R. Yunta, R. Costa and E. Orti, Org. Lett., 2008, 10, 5099-5102. 
5 S. W. Thomas and T. M. Swager, Adv. Mater., 2006, 18, 10471050.

6 H. Nohta, H. Satozono, K. Koiso, H. Yoshida, J. Ishida and M. Yamaguchi, Anal. Chem., 2000, 72, 4199-4204.

7 K. I. Oberg, R. Hodyss and J. L. Beauchamp, Sens. Actuators, $B, 2006,115,79-85$.

8 Y. Jin, J. W. Jang, M. H. Lee and C. H. Han, Clin. Chim. Acta, 2006, 364, 260-266.

9 W. Qin, P. Parzuchowski, W. Zhang and M. E. Meyerhoff, Anal. Chem., 2003, 75, 332-340.

10 J. M. Charlesworth and C. A. McDonald, Sens. Actuators, B, 1992, 8, 137-142.

11 M. McCarrick, S. J. Harris and D. Diamond, J. Mater. Chem., 1994, 4, 217-221.

12 T. M. Swager, Acc. Chem. Res., 2008, 41, 1181-1189.

13 R. Ziessel, G. Ulrich, A. Harriman, M. A. Alamiry, B. Stewart and P. Retailleau, Chem.-Eur. J., 2009, 15, 1359-1369.

14 H. Y. Xia, G. He, J. X. Peng, W. W. Li and Y. Fang, Appl. Surf. Sci., 2010, 256, 7270-7275.

15 H. Y. Xia, G. He, L. N. Gao, J. X. Peng and Y. Fang, Chem. J. Chin. Univ., 2010, 31, 1614-1619.

16 H. N. Peng, L. P. Ding, T. H. Liu, X. L. Chen, L. Li, S. W. Yin and Y. Fang, Chem.-Asian J., 2012, 7, 1576-1582.

17 L. Dong, C. M. Deng, C. He, L. Shib, Y. Y. Fu, D. F. Zhu, H. M. Cao, Q. G. He and J. G. Cheng, Sens. Actuators, B, 2013, 180, 28-34.

18 Y. M. Chung, B. Raman and K. H. Ahn, Tetrahedron, 2006, 62, 11645-11651.

19 A. Satrijo and T. M. Swager, J. Am. Chem. Soc., 2007, 12, 16020-16028.

20 T. M. Swager, Acc. Chem. Res., 2008, 41, 1181-1189.

21 S. W. Thomas, G. D. Joly and T. M. Swager, Chem. Rev., 2007, 107, 1339-1386.

22 U. K. Gautam, X. S. Fang, Y. Bando, J. H. Zhan and D. Golberg, ACS Nano, 2008, 2, 1015-1021.

23 X. S. Fang, Y. Bando, U. K. Gautam, C. H. Ye and D. Golberg, J. Mater. Chem., 2008, 18, 509-522.

24 H. Liu, L. F. Hu, K. Watanabe, X. H. Hu, B. Dierre, B. Kim, T. Sekiguchi and X. S. Fang, Adv. Funct. Mater., 2013, 23, 3701-3709.

25 A. K. Giria, C. Charanb, S. C. Ghosha, V. K. Shahib and A. B. Panda, Sens. Actuators, B, 2016, 229, 14-24.

26 C. Y. Zhou, Z. F. Wu, Y. N. Guo, Y. S. Li, H. Y. Cao, X. F. Zheng and X. C. Dou, Sci. Rep., 2016, 6, 25588, DOI: 10.1038/ srep25588.

27 X. Fang, L. Wu and L. Hu, Adv. Mater., 2011, 23, 585-598.

28 R. G. Zhang, B. Y. Wang and L. Wei, Mater. Chem. Phys., 2008, 112, 557-561.

29 T. Y. Zhai, Z. J. Hu, Y. Ma, W. S. Yang, L. Y. Zhao and J. N. Yao, Mater. Chem. Phys., 2006, 100, 281-284.

30 X. J. Xu, G. T. Fei, W. H. Yu, X. W. Wang, L. Chen and L. D. Zhang, Nanotechnology, 2006, 17, 426-429.

31 M. Yokoyama, K. i. Kashiro and S. i. Ohta, J. Cryst. Growth, 1987, 81, 73-78.

32 Z. G. Chen, L. Cheng, J. Zou, X. D. Yao, G. Q. Lu and H. Cheng, Nanotechnology, 2010, 21, 065701.
33 G. X. Qian, K. F. Hu, J. J. Fu and P. K. Chu, J. Nanosci. Nanotechnol., 2009, 9, 3347-3351.

34 K. Murata, M. Aoki, T. Suzuki, T. Harada, H. Kawabata, T. Komori, F. Ohseto, K. Ueda and S. Shinkai, J. Am. Chem. Soc., 1994, 116, 6664-6676.

35 S. Shinkai and K. Murata, J. Mater. Chem., 1998, 8, 485-495.

36 K. Yoza, N. Amanokura, Y. Ono, T. Akao, H. Shinmori, M. Takeuchi, S. Shinkai and D. L. Reinhout, Chem.-Eur. J., 1999, 5, 2722-2729.

37 K. J. C Bommel, A. Friggeri and S. Shinkai, Angew. Chem., Int. Ed. Engl., 2003, 42, 980-999.

38 M. George and R. G. Weiss, Acc. Chem. Res., 2006, 39, 489497.

39 K. Jyothish, M. Hariharan and D. Ramaiah, Chem.-Eur. J., 2007, 13, 5944-5951.

40 H. Y. Xia, J. X. Peng, K. Q. Liu, C. Li and Y. Fang, J. Phys. D: Appl. Phys., 2008, 41, 105405.

41 Z. Zou, Y. Qiu, C. Xie, J. Xu, Y. Luo, C. Wang and H. Yan, J. Alloys Compd., 2015, 645, 17-23.

42 K. Xu, N. Li, D. Zeng, S. Tian, S. Zhang, D. Hu and C. Xie, ACS Appl. Mater. Interfaces, 2015, 7, 11359-11368.

43 A. Gaiardo, B. Fabbri, V. Guidi, P. Bellutti, A. Giberti, S. Gherardi, L. Vanzetti, C. Malagù and G. Zonta, Sensors, 2016, 16, 296.

44 X. Fu, J. Liu, Y. Wan, X. Zhang, F. Meng and J. Liu, J. Mater. Chem., 2012, 22, 17782-17791.

45 M. M. Haque, H. Im, J. Seo, M. Hasan, K. Wood and O. Kwona, J. Appl. Toxicol., 2013, 33, 940-950.

46 S. T. Hossain and S. K. Mukherjee, J. Hazard. Mater., 2013, 260, 1073-1082.

47 J. X. Peng, K. Q. Liu, J. Liu, Q. H. Zhang, X. L. Feng and Y. Fang, Langmuir, 2008, 24, 2992-3000.

48 J. S. Chen, K. Žídek, M. Abdellah, M. J. Al-Marri, K. Zheng and T. Pullerits, J. Phys.: Condens. Matter, 2016, 28, 254001.

49 G. Murugadoss, J. Lumin., 2012, 132, 2043-2048.

50 N. Kumbhojkar, V. V. Nikesh, A. Kshirsagar and S. Mahamuni, J. Appl. Phys., 2000, 88, 6260-6264.

51 J. W. M. Chon, M. Gu, C. Bullen and P. Mulvaney, Appl. Phys. Lett., 2004, 84, 472-4474.

52 S. Santhi, E. Bernstein and F. Paille, J. Lumin., 2006, 117, 101-112.

53 M. V. Limaye, S. Gokhale, S. A. Acharya and S. K. Kulkarni, Nanotechnology, 2008, 19, 415602.

54 A. L. Donne, S. K. Jana, S. Banerjee, S. Basu and S. Binetti, J. Appl. Phys., 2013, 113, 014903.

55 W. Chen, Z. G. Wang, Z. J. Lin and L. Y. Lin, J. Appl. Phys., 1997, 82, 3111-3115.

56 G. C. Lisensky, R. L. Penn, C. J. Murphy and A. B. Ellis, Science, 1990, 248, 840.

57 H. Y. Du, G. He, T. H. Liu, L. P. Ding and Y. Fang, J. Photochem. Photobiol., A, 2010, 217, 356-362.

58 J. R. Lakowicz, Principles of Fluorescence Spectroscopy, Kluwer/Plenum, New York, 2nd edn, 1999.

59 C. F. Landes, M. Braun and M. A. El-Sayed, J. Phys. Chem. B, 2001, 105, 10554-10558.

60 C. Landes, C. Burda, M. Braun and M. A. El-Sayed, J. Phys. Chem. B, 2001, 105, 2981-2986. 